\title{
Best possible inequalities for the harmonic mean of error function
}

\author{
Yu-Ming Chu' ${ }^{1}$ Yong-Min Li ${ }^{2}$, Wei-Feng Xia ${ }^{1 *}$ and Xiao-Hui Zhang ${ }^{1}$
}

\author{
"Correspondence: xwf212@163.com \\ ${ }^{1}$ School of Mathematics and \\ Computation Sciences, Hunan City \\ University, Yiyang, 413000, China \\ Full list of author information is \\ available at the end of the article
}

\begin{abstract}
In this paper, we find the least value $r$ and the greatest value $p$ such that the double inequality $\operatorname{erf}\left(M_{p}(x, y ; \lambda)\right) \leq H(\operatorname{erf}(x), \operatorname{erf}(y) ; \lambda) \leq \operatorname{erf}\left(M_{r}(x, y ; \lambda)\right)$ holds for all $x, y \geq 1$ (or $0<x, y<1)$ with $0<\lambda<1$, where $\operatorname{erf}(x)=\frac{2}{\sqrt{\pi}} \int_{0}^{x} e^{-t^{2}} d t$, and $M_{p}(x, y ; \lambda)=\left(\lambda x^{p}+(1-\lambda) y^{p}\right)^{1 / p}(p \neq 0)$ and $M_{0}(x, y ; \lambda)=x^{\lambda} y^{1-\lambda}$ are, respectively, the error function, and weighted power mean.
\end{abstract}

MSC: 33B20; 26D15

Keywords: error function; power mean; functional inequalities

\section{Introduction}

For $x \in R$, the error function $\operatorname{erf}(x)$ is defined as

$$
\operatorname{erf}(x)=\frac{2}{\sqrt{\pi}} \int_{0}^{x} e^{-t^{2}} d t
$$

It is well known that the error function $\operatorname{erf}(x)$ is odd, strictly increasing on $(-\infty,+\infty)$ with $\lim _{x \rightarrow+\infty} \operatorname{erf}(x)=1$, strictly concave and strictly log-concave on $[0,+\infty)$. For the $n$th derivation we have the representation

$$
\frac{d^{n}}{d x^{n}} \operatorname{erf}(x)=(-1)^{n-1} \frac{2}{\sqrt{\pi}} e^{-x^{2}} H_{n-1}(x)
$$

where $H_{n}(x)=(-1)^{n} e^{x^{2}} \frac{d^{n}}{d x^{n}}\left(e^{-x^{2}}\right)$ is a Hermite polynomial.

The error function can be expanded as a power series in the following two ways [1]:

$$
\operatorname{erf}(x)=\frac{2}{\sqrt{\pi}} \sum_{n=0}^{+\infty} \frac{(-1)^{n}}{n !(2 n+1)} x^{2 n+1}=e^{-x^{2}} \sum_{n=0}^{+\infty} \frac{1}{\Gamma\left(n+\frac{3}{2}\right)} x^{2 n+1} .
$$

It also can be expressed in terms of incomplete gamma function and a confluent hypergeometric function:

$$
\operatorname{erf}(x)=\frac{\operatorname{sgn}(x)}{\sqrt{\pi}} \gamma\left(\frac{1}{2}, x^{2}\right)=\frac{2 x}{\sqrt{\pi}}{ }_{1} F_{1}\left(\frac{1}{2} ; \frac{3}{2} ;-x^{2}\right)
$$

Recently, the error function have been the subject of intensive research. In particular, many remarkable properties and inequalities for the error function can be found in the

\section{Springer}

๑2014 Chu et al.; licensee Springer. This is an Open Access article distributed under the terms of the Creative Commons Attribution License (http://creativecommons.org/licenses/by/2.0), which permits unrestricted use, distribution, and reproduction in any medium, provided the original work is properly cited. 
literature [2-10]. It might be surprising that the error function has applications in heat conduction problems $[11,12]$.

In [13], Chu proved that the double inequality

$$
\sqrt{1-e^{-a x^{2}}} \leq \operatorname{erf}(x) \leq \sqrt{1-e^{-b x^{2}}}
$$

holds for all $x \geq 0$ if and only if $0 \leq a \leq 1$ and $b \geq \frac{4}{\pi}$.

Mitrinović and Weinacht [14] established that

$$
\operatorname{erf}(x)+\operatorname{erf}(y) \leq \operatorname{erf}(x+y)+\operatorname{erf}(x) \operatorname{erf}(y)
$$

for all $x, y \geq 0$, and proved that the inequality become equality if and only if $x=0$ or $y=0$.

In $[15,16]$ Alzer proved that

$$
\alpha_{n}=\left\{\begin{array}{ll}
0.90686 \cdots, & \text { if } n=2, \\
1, & \text { if } n \geq 3
\end{array} \text { and } \quad \beta_{n}=n-1\right.
$$

are the best possible constants such that the double inequality

$$
\alpha_{n} \operatorname{erf}\left(\sum_{i=1}^{n} x_{i}\right) \leq \sum_{i=1}^{n} \operatorname{erf}\left(x_{i}\right)-\prod_{i=1}^{n} \operatorname{erf}\left(x_{i}\right) \leq \beta_{n} \operatorname{erf}\left(\sum_{i=1}^{n} x_{i}\right)
$$

holds for $n \geq 2$ and all real number $x_{i} \geq 0(i=1,2, \ldots, n)$, and the sharp double inequalities

$$
\operatorname{erf}(1)<\frac{\operatorname{erf}(x+\operatorname{erf}(y))}{\operatorname{erf}(y+\operatorname{erf}(x))}<\frac{2}{\sqrt{\pi}}
$$

and

$$
0<\frac{\operatorname{erf}(x \operatorname{erf}(y))}{\operatorname{erf}(y \operatorname{erf}(x))} \leq 1
$$

hold for all positive real numbers $x, y$ with $x \geq y$.

Let $\lambda \in(0,1)$, and $A(x, y ; \lambda)=\lambda x+(1-\lambda) y, G(x, y ; \lambda)=x^{\lambda} y^{1-\lambda}, H(x, y ; \lambda)=x y /[\lambda y+(1-\lambda) x]$, and $M_{r}(x, y ; \lambda)=\left[\lambda x^{r}+(1-\lambda) y^{r}\right]^{1 / r}(r \neq 0)$ and $M_{0}(x, y ; \lambda)=x^{\lambda} y^{1-\lambda}$ be, respectively, the weighted arithmetic, geometric, harmonic, and power means of two positive numbers $x$ and $y$. Then it is well known that the inequalities

$$
H(x, y ; \lambda)=M_{-1}(x, y ; \lambda)<G(x, y ; \lambda)=M_{0}(x, y ; \lambda)<A(x, y ; \lambda)=M_{1}(x, y ; \lambda)
$$

hold for all $\lambda \in(0,1)$ and $x, y>0$ with $x \neq y$.

Very recently, Alzer [17] proved that $c_{1}(\lambda)=[\lambda+(1-\lambda) \operatorname{erf}(1)] /[\operatorname{erf}(1 /(1-\lambda))]$ and $c_{2}(\lambda)=1$ are the best possible factors such that the double inequality

$$
c_{1}(\lambda) \operatorname{erf}(H(x, y ; \lambda)) \leq A(\operatorname{erf}(x), \operatorname{erf}(y) ; \lambda) \leq c_{2}(\lambda) \operatorname{erf}(H(x, y ; \lambda))
$$

holds for all $x, y \in[1,+\infty)$ and $\lambda \in(0,1 / 2)$. 
It is natural to ask what are the least value $r$ and the greatest value $p$ such that the double inequality

$$
\operatorname{erf}\left(M_{p}(x, y ; \lambda)\right) \leq H(\operatorname{erf}(x), \operatorname{erf}(y) ; \lambda) \leq \operatorname{erf}\left(M_{r}(x, y ; \lambda)\right)
$$

holds for all $x, y \geq 1$ (or $0<x, y<1$ )? The main purpose of this article is to answer this question.

\section{Lemmas}

In order to prove our main results we need three lemmas, which we present in this section.

Lemma 2.1 Let $r \neq 0$ and $J(x)=\frac{1}{r}\left[\operatorname{erf}\left(x^{1 / r}\right)-\frac{1}{\sqrt{\pi}} x^{1 / r} e^{-x^{2 / r}}\right]$. Then the following statements are true:

(1) if $-1 \leq r<0$, then $J(x)<0$ for all $x \in(0,+\infty)$;

(2) if $0<r<1$, then $J(x)>0$ for all $x \in(0,+\infty)$.

Proof Simple computation leads to

$$
J^{\prime}(x)=\frac{1}{r^{2}} \frac{2}{\sqrt{\pi}} x^{1 / r-1} e^{-x^{2 / r}}\left(\frac{1}{2}+x^{2 / r}\right)>0
$$

for all $x \in(0,+\infty)$.

(1) If $-1 \leq r<0$, then we clearly see that

$$
\lim _{x \rightarrow+\infty} J(x)=0 .
$$

Therefore, Lemma 2.1(1) follows easily from (2.1) and (2.2).

(2) If $0<r<1$, then it is obvious that

$$
\lim _{x \rightarrow 0^{+}} J(x)=0 .
$$

Therefore, Lemma 2.1(2) follows from (2.1) and (2.3).

Lemma 2.2 Let $r \neq 0, r_{0}=-1-\frac{4}{e \sqrt{\pi} \operatorname{erf}(1)}=-1.9852 \cdots$ and $u(x)=\frac{1}{\operatorname{erf}\left(x^{1 / r}\right)}$. Then the following statements are true:

(1) if $r \leq r_{0}$, then $u(x)$ is strictly concave on $[1,+\infty)$;

(2) if $r_{0} \leq r<-1$, then $u(x)$ is strictly convex on $(0,1]$;

(3) if $r \geq-1$, then $u(x)$ is strictly convex on $(0,+\infty)$.

Proof Differentiating $u(x)$ leads to

$$
u^{\prime}(x)=-\frac{1}{r} \frac{x^{1 / r-1} \operatorname{erf}^{\prime}\left(x^{1 / r}\right)}{\operatorname{erf}^{2}\left(x^{1 / r}\right)}
$$

and

$$
u^{\prime \prime}(x)=\frac{1}{r^{2}} \frac{2}{\sqrt{\pi}} \frac{1}{\operatorname{erf}^{2}\left(x^{1 / r}\right)} x^{1 / r-2} e^{-x^{2 / r}} g(x),
$$


where

$$
g(x)=\left(r-1+2 x^{2 / r}\right) \operatorname{erf}\left(x^{1 / r}\right)+\frac{4}{\sqrt{\pi}} x^{1 / r} e^{-x^{2 / r}} .
$$

It follows from (2.6) that

$$
\begin{aligned}
& g(1)=(r+1) \operatorname{erf}(1)+\frac{4}{e \sqrt{\pi}}, \\
& g^{\prime}(x)=4 x^{2 / r-1} g_{1}(x), \\
& g_{1}(x)=\frac{1}{r} \operatorname{erf}\left(x^{1 / r}\right)+\frac{1}{r} \frac{1}{2 \sqrt{\pi}} x^{1 / r}\left[(1+r) x^{-2 / r}-2\right] e^{-x^{2 / r}}, \\
& g_{1}^{\prime}(x)=\frac{1}{r^{2}} \frac{1}{2 \sqrt{\pi}} x^{-1 / r-1} e^{-x^{2 / r}} g_{2}(x), \\
& g_{2}(x)=4 x^{4 / r}-2 r x^{2 / r}-(1+r) .
\end{aligned}
$$

We divide the proof into four cases.

Case $1 r<-1$. Then from (2.6) and (2.8) together with (2.9) we clearly see that

$$
\begin{aligned}
& \lim _{x \rightarrow 0^{+}} g(x)=+\infty, \quad \lim _{x \rightarrow+\infty} g(x)=0, \\
& \lim _{x \rightarrow 0^{+}} g_{1}(x)=\frac{1}{r}<0, \quad \lim _{x \rightarrow+\infty} g_{1}(x)=+\infty, \\
& \lim _{x \rightarrow+\infty} g_{2}(x)=-(1+r)>0,
\end{aligned}
$$

and $g_{2}(x)$ is strictly decreasing on $[0,+\infty)$.

It follows from the monotonicity of $g_{2}(x)$ and (2.12) that $g_{1}(x)$ is strictly increasing on $[0,+\infty)$.

The monotonicity of $g_{1}(x)$ and $(2.11)$ imply that there exists $x_{1} \in(0,+\infty)$, such that $g_{1}(x)<$ 0 for $x \in\left(0, x_{1}\right)$ and $g_{1}(x)>0$ for $x \in\left(x_{1},+\infty\right)$. Therefore, $g(x)$ is strictly decreasing on $\left[0, x_{1}\right]$ and strictly increasing on $\left[x_{1},+\infty\right)$.

From the piecewise monotonicity of $g(x)$ and (2.10) we clearly see that there exists $x_{2} \in$ $(0,+\infty)$, such that $g(x)>0$ for $x \in\left(0, x_{2}\right)$ and $g(x)<0$ for $x \in\left(x_{2},+\infty\right)$.

If $r \leq r_{0}$, then (2.7) leads to $g(1) \leq 0$, this implies that $g(x)<0$ for $x \in(1,+\infty)$. Therefore, (2.5) leads to the conclusion that $u(x)$ is strictly concave on $[1,+\infty)$.

If $r_{0} \leq r<-1$, then (2.7) leads to $g(1) \geq 0$, this implies that $g(x)>0$ for $x \in(0,1)$. Therefore, (2.5) leads to the conclusion that $u(x)$ is strictly convex on $(0,1)$.

Case $2-1 \leq r<0$. Then we clearly see that the function $(1+r) x^{-2 / r}-2$ is strictly increasing on $(0,+\infty)$ with $\lim _{x \rightarrow 0^{+}}\left[(1+r) x^{-2 / r}-2\right]=-2$, and

$$
g_{1}(x)<\frac{1}{r}\left[\operatorname{erf}\left(x^{1 / r}\right)-\frac{1}{\sqrt{\pi}} x^{1 / r} e^{-x^{2 / r}}\right]
$$

Therefore, Lemma 2.1(1) and (2.13) imply that $g_{1}(x)<0$ for $x \in(0,+\infty)$. This leads to the conclusion that $g(x)$ is strictly decreasing on $(0,+\infty)$. 
From (2.6) we get

$$
\lim _{x \rightarrow+\infty} g(x)=0
$$

for $-1 \leq r<0$.

It follows from the monotonicity of $g(x)$ and (2.14) that $g(x)>0$ for $x \in(0,+\infty)$. Therefore, $(2.5)$ leads to the conclusion that $u(x)$ is strictly convex on $(0,+\infty)$.

Case $30<r<1$. Then we clearly see that the function $(1+r) x^{-2 / r}-2$ is strictly decreasing on $(0,+\infty)$ with $\lim _{x \rightarrow+\infty}\left[(1+r) x^{-2 / r}-2\right]=-2$, and

$$
g_{1}(x)>\frac{1}{r}\left[\operatorname{erf}\left(x^{1 / r}\right)-\frac{1}{\sqrt{\pi}} x^{1 / r} e^{-x^{2 / r}}\right] .
$$

It follows from Lemma 2.1(2) and (2.15) that $g_{1}(x)>0$ for $x \in(0,+\infty)$. This leads to $g(x)$ being strictly increasing on $(0,+\infty)$.

It follows from (2.6) that

$$
\lim _{x \rightarrow 0^{+}} g(x)=0
$$

for $0<r<1$.

From the monotonicity of $g(x)$ and (2.16) we know that $g(x)>0$ for $x \in(0,+\infty)$. Therefore, (2.5) leads to the conclusion that $u(x)$ is strictly convex on $(0,+\infty)$.

Case $4 r \geq 1$. Then from (2.6) we clearly see that $g(x)>0$ for $x \in(0,+\infty)$. Therefore, $u(x)$ is strictly convex on $(0,+\infty)$ follows easily from $(2.5)$.

Lemma 2.3 The function $h(x)=x^{2}+\frac{x e^{-x^{2}}}{\int_{0}^{x} e^{-t^{2}} d t}$ is strictly increasing on $(0,+\infty)$.

Proof Simple computations lead to

$$
h^{\prime}(x)=\frac{h_{1}(x)}{\left(\int_{0}^{x} e^{-t^{2}} d t\right)^{2}},
$$

where

$$
\begin{aligned}
& h_{1}(x)=2 x\left(\int_{0}^{x} e^{-t^{2}} d t\right)^{2}+\left(1-2 x^{2}\right) e^{-x^{2}} \int_{0}^{x} e^{-t^{2}} d t-x e^{-2 x^{2}}, \\
& h_{1}(0)=0, \quad h_{1}(1)=0.7054 \cdots>0 \\
& h_{1}^{\prime}(x)=2\left(\int_{0}^{x} e^{-t^{2}} d t\right)^{2}+2 x\left(2 x^{2}-1\right) e^{-x^{2}} \int_{0}^{x} e^{-t^{2}} d t+2 x^{2} e^{-2 x^{2}}, \\
& h_{1}^{\prime}(0)=0 \\
& h_{1}^{\prime \prime}(x)=e^{-x^{2}} h_{2}(x), \\
& h_{2}(x)=\left(-8 x^{4}+16 x^{2}+2\right) \int_{0}^{x} e^{-t^{2}} d t+\left(-4 x^{3}+2 x\right) e^{-x^{2}}, \\
& h_{2}(0)=0 \\
& h_{2}^{\prime}(x)=32 x\left(1-x^{2}\right) \int_{0}^{x} e^{-t^{2}} d t+4 e^{-x^{2}} .
\end{aligned}
$$


We divide the proof into two cases.

Case $1 x \geq 1$. Then (2.19) leads to $h_{1}^{\prime}(x)>0$. Therefore, $h^{\prime}(x)>0$ follows from (2.18) and (2.17)

Case $20<x<1$. Then from (2.23) we clearly see that $h_{2}^{\prime}(x)>0$. Therefore, $h^{\prime}(x)>0$ follows from (2.17) and (2.18) together with (2.20)-(2.22).

\section{Main results}

Theorem 3.1 Let $\lambda \in(0,1)$ and $r_{0}=-1-\frac{4}{e \sqrt{\pi} \operatorname{erf}(1)}=-1.9852 \cdots$. Then the double inequality

$$
\operatorname{erf}\left(M_{\mu}(x, y ; \lambda)\right) \leq H(\operatorname{erf}(x), \operatorname{erf}(y) ; \lambda) \leq \operatorname{erf}\left(M_{\nu}(x, y ; \lambda)\right)
$$

holds for all $0<x, y<1$ if and only if $\mu \leq r_{0}$ and $v \geq-1$.

Proof Firstly, we prove that (3.1) holds if $\mu \leq r_{0}$ and $v \geq-1$.

If $\mu \leq r_{0}, u(z)=\frac{1}{\operatorname{erf}\left(z^{1 / \mu}\right)}$, then Lemma 2.2(1) leads to

$$
\lambda u(s)+(1-\lambda) u(t) \leq u(\lambda s+(1-\lambda) t)
$$

for $\lambda \in(0,1)$ and $s, t>1$.

Let $s=x^{\mu}, t=y^{\mu}$, and $0<x, y<1$. Then (3.2) leads to the first inequality in (3.1).

Since the function $t \mapsto \operatorname{erf}\left(M_{t}(x, y ; \lambda)\right)$ is strictly increasing on $R$ if $v \geq-1$, it is enough to prove the second inequality in (3.1) is true for $-1 \leq v<0$.

Let $-1 \leq v<0$ and $u(z)=\frac{1}{\operatorname{erf}\left(z^{1 / v}\right)}$. Then Lemma 2.2(3) leads to

$$
u(\lambda s+(1-\lambda) t) \leq \lambda u(s)+(1-\lambda) u(t)
$$

for $\lambda \in(0,1)$ and $s, t>1$.

Therefore, the second inequality in (3.1) follows from $s=x^{v}$ and $t=y^{v}$ together with (3.3).

Secondly, we prove that the second inequality in (3.1) implies $v \geq-1$.

Let $0<x, y<1$. Then the second inequality in (3.1) leads to

$$
D(x, y):=\operatorname{erf}\left(M_{v}(x, y ; \lambda)\right)-H(\operatorname{erf}(x), \operatorname{erf}(y) ; \lambda) \geq 0 .
$$

It follows from (3.4) that

$$
D(y, y)=\left.\frac{\partial}{\partial x} D(x, y)\right|_{x=y}=0
$$

and

$$
\left.\frac{\partial^{2}}{\partial x^{2}} D(x, y)\right|_{x=y}=\lambda(1-\lambda) y^{-1} \operatorname{erf}^{\prime}(y)\left[v-1+2\left(y^{2}+\frac{y e^{-y^{2}}}{\int_{0}^{y} e^{-t^{2}} d t}\right)\right] .
$$

Therefore,

$$
v \geq \lim _{y \rightarrow 0^{+}}\left[1-2\left(y^{2}+\frac{y e^{-y^{2}}}{\int_{0}^{y} e^{-t^{2}} d t}\right)\right]=-1
$$

follows from (3.4) and (3.5) together with Lemma 2.3. 
Finally, we prove that the first inequality in (3.1) implies $\mu \leq r_{0}$.

Let $y \rightarrow 1$. Then the first inequality in (3.1) leads to

$$
L(x)=: H(\operatorname{erf}(x), \operatorname{erf}(1) ; \lambda)-\operatorname{erf}\left(m_{\mu}(x, 1 ; \lambda)\right) \geq 0
$$

for $0<x<1$.

It follows from (3.6) that

$$
\begin{aligned}
& L(1)=0, \\
& {[\lambda \operatorname{erf}(1)+(1-\lambda) \operatorname{erf}(x)]^{2} L^{\prime}(x)=\frac{2 \lambda}{\sqrt{\pi}} e^{-x^{2}} L_{1}(x),}
\end{aligned}
$$

where

$$
\begin{aligned}
& L_{1}(x)=\operatorname{erf}(1)^{2}-x^{\mu-1}\left(\lambda x^{\mu}+1-\lambda\right)^{\frac{1}{\mu}-1}[\lambda \operatorname{erf}(1)+(1-\lambda) \operatorname{erf}(x)]^{2} e^{x^{2}-\left(\lambda x^{\mu}+1-\lambda\right)^{\frac{2}{\mu}},} \\
& \lim _{x \rightarrow 1^{-}} L_{1}(x)=0, \\
& \lim _{x \rightarrow 1^{-}} L_{1}^{\prime}(x)=(1-\lambda) \operatorname{erf}(1)^{2}\left[-1-\mu-\frac{4}{e \sqrt{\pi} \operatorname{erf}(1)}\right] .
\end{aligned}
$$

If $\mu>r_{0}$, then from (3.10) we know that there exists a small $\delta_{1}>0$, such that $L_{1}^{\prime}(x)<0$ for $x \in\left(1-\delta_{1}, 1\right)$. Therefore, $L_{1}(x)$ is strictly decreasing on $\left[1-\delta_{1}, 1\right]$.

The monotonicity of $L_{1}(x)$ together with (3.8) and (3.9) imply that there exists $\delta_{2}>0$, such that $L(x)$ is strictly increasing on $\left(1-\delta_{2}, 1\right)$

It follows from the monotonicity of $L(x)$ and (3.7) that there exists $\delta_{3}>0$, such that $L(x)<0$ for $x \in\left(1-\delta_{3}, 1\right)$, this contradicts with (3.6).

Theorem 3.2 Let $\lambda \in(0,1)$ and $r_{0}=-1-\frac{4}{e \sqrt{\pi} \operatorname{erf}(1)}=-1.9852 \cdots$. Then the double inequality

$$
\operatorname{erf}\left(M_{p}(x, y ; \lambda)\right) \leq H(\operatorname{erf}(x), \operatorname{erf}(y) ; \lambda) \leq \operatorname{erf}\left(M_{r}(x, y ; \lambda)\right)
$$

holds for all $x, y \geq 1$ if and only if $p=-\infty$ and $r \geq r_{0}$.

Proof Firstly, we prove that inequality (3.11) holds if $p=-\infty$ and $r \geq r_{0}$. Since the first inequality in (3.11) is true if $p=-\infty$, thus we only need to prove the second inequality in (3.11).

It follows from the monotonicity of the function $\operatorname{erf}\left(M_{t}(x, y ; \lambda)\right)$ with respect to $t$ that we only need to prove the second inequality in (3.11) holds for $r_{0} \leq r<-1$.

Let $r_{0} \leq r<-1$ and $u(z)=\frac{1}{\operatorname{erf}\left(z^{1 / r}\right)}$. Then Lemma 2.2(2) leads to

$$
u(\lambda s+(1-\lambda) t) \leq \lambda u(s)+(1-\lambda) u(t)
$$

for $\lambda \in(0,1)$ and $s, t \in(0,1]$.

Therefore, the second inequality in (3.11) follows from $s=x^{r}$ and $t=y^{r}$ together with (3.12).

Secondly, we prove that the second inequality in (3.11) implies $r \geq r_{0}$. 
Let $x \geq 1$ and $y \geq 1$. Then the second inequality in (3.11) leads to

$$
K(x, y)=: \operatorname{erf}\left(M_{r}(x, y ; \lambda)\right)-H(\operatorname{erf}(x), \operatorname{erf}(y) ; \lambda) \geq 0 .
$$

It follows from (3.13) that

$$
K(y, y)=\left.\frac{\partial}{\partial x} K(x, y)\right|_{x=y}=0
$$

and

$$
\left.\frac{\partial^{2}}{\partial x^{2}} K(x, y)\right|_{x=y}=\lambda(1-\lambda) y^{-1} \operatorname{erf}^{\prime}(y)\left[r-1+2\left(y^{2}+\frac{y e^{-y^{2}}}{\int_{0}^{y} e^{-t^{2}} d t}\right)\right] .
$$

Therefore,

$$
r \geq \lim _{y \rightarrow 1^{+}}\left[1-2\left(y^{2}+\frac{y e^{-y^{2}}}{\int_{0}^{y} e^{-t^{2}} d t}\right)\right]=r_{0}
$$

follows from (3.13) and (3.14) together with Lemma 2.3.

Finally, we prove that the first inequality in (3.11) implies $p=-\infty$. We divide the proof into two cases.

Case $1 p \geq 0$. Then for any fixed $y \in(1,+\infty)$ one has

$$
\lim _{x \rightarrow+\infty} \operatorname{erf}\left(M_{p}(x, y ; \lambda)\right)=1
$$

and

$$
\lim _{x \rightarrow+\infty} H(\operatorname{erf}(x), \operatorname{erf}(y) ; \lambda)=\frac{\operatorname{erf}(y)}{\lambda \operatorname{erf}(y)+1-\lambda}<1,
$$

which contradicts with the first inequality in (3.11).

Case $2-\infty<p<0$. Let $x \geq 1, \theta=\lambda^{1 / p}$, and $y \rightarrow+\infty$. Then the first inequality in (3.11) leads to

$$
T(x)=: H(\operatorname{erf}(x), 1 ; \lambda)-\operatorname{erf}(\theta x) \geq 0 .
$$

It follows from (3.15) that

$$
\lim _{x \rightarrow+\infty} T(x)=0
$$

and

$$
[\lambda+(1-\lambda) \operatorname{erf}(x)]^{2} T^{\prime}(x)=\frac{2}{\sqrt{\pi}} e^{-x^{2}}\left[\lambda-(\lambda+(1-\lambda) \operatorname{erf}(x))^{2} \theta e^{\left(1-\theta^{2}\right) x^{2}}\right] .
$$

Note that

$$
\lim _{x \rightarrow+\infty}\left[\lambda-(\lambda+(1-\lambda) \operatorname{erf}(x))^{2} \theta e^{\left(1-\theta^{2}\right) x^{2}}\right]=\lambda>0
$$


It follows from (3.17) and (3.18) that there exists a large enough $\eta_{1} \in(0,+\infty)$, such that $T^{\prime}(x)>0$ for $x \in\left(\eta_{1},+\infty\right)$, hence $T(x)$ is strictly increasing on $\left[\eta_{1},+\infty\right)$.

From the monotonicity of $T(x)$ and (3.16) we conclude that there exists a large enough $\eta_{2} \in(0,+\infty)$, such that $T(x)<0$ for $x \in\left(\eta_{2},+\infty\right)$, this contradicts with (3.15).

\section{Competing interests}

The authors declare that they have no competing interests.

\section{Authors' contributions}

All authors contributed equally to the writing of this paper. All authors read and approved the final manuscript.

\section{Author details}

${ }^{1}$ School of Mathematics and Computation Sciences, Hunan City University, Yiyang, 413000, China. ${ }^{2}$ Department of Mathematics, Huzhou University, Huzhou, 313000, China.

\section{Acknowledgements}

This research was supported by the Natural Science Foundation of China under Grants 61174076, 61374086, 11371125, and 11401191, and the Natural Science Foundation of Zhejiang Province under Grant LY13A010004. The authors wish to thank the anonymous referees for their careful reading of the manuscript and their fruitful comments and suggestions.

\section{Received: 8 October 2014 Accepted: 11 December 2014 Published: 23 Dec 2014}

\section{References}

1. Oldham, K, Myland, J, Spanier, J: An Atlas of Functions. Springer, New York (2009)

2. Alzer, H, Baricz, Á: Functional inequalities for the incomplete gamma function. J. Math. Anal. Appl. 385(1), 167-178 (2012)

3. Baricz, Á: Mills' ratio: monotonicity patterns and functional inequalities. J. Math. Anal. Appl. 340(2), 1362-1370 (2008)

4. Dominici, D: Some properties of the inverse error function. Contemp. Math. 457, 191-203 (2008)

5. Gawronski, W, Müller, J, Reinhard, M: Reduced cancellation in the evaluation of entire functions and applications to the error function. SIAM J. Numer. Anal. 45(6), 2564-2576 (2007)

6. Morosawa, S: The parameter space of error functions of the form a $\int_{0}^{z} e^{-w^{2}} d w$. In: Complex Analysis and Potential Theory, pp. 174-177. World Scientific, Hackensack (2007)

7. Fisher, B, Al-Sirehy, F, Telci, M: Convolutions involving the error function. Int. J. Appl. Math. 13(4), $317-326$ (2003)

8. Alzer, H: On some inequalities for the incomplete gamma function. Math. Comput. 66(218), 771-778 (1997)

9. Chapman, SJ: On the non-universality of the error function in the smoothing of Stokes discontinuities. Proc. R. Soc. Lond. Ser. A 452(1953), 2225-2230 (1996)

10. Weideman, JAC: Computation of the complex error function. SIAM J. Numer. Anal. 31(5), 1497-1518 (1994)

11. Kharin, SN: A generalization of the error function and its application in heat conduction problems. In: Differential Equations and Their Applications, vol. 176, pp. 51-59. 'Nauka' Kazakh. SSR, Alma-Ata (1981) (in Russian)

12. Chaudhry, MA, Qadir, A, Zubair, SM: Generalized error functions with applications to probability and heat conduction. Int. J. Appl. Math. 9(3), 259-278 (2002)

13. Chu, JT: On bounds for the normal integral. Biometrika 42, 263-265 (1955)

14. Mitrinović, DS, Weinacht, RJ: Problems and solutions: solutions of advanced problems: 5555. Am. Math. Mon. 75(10), 1129-1130 (1968)

15. Alzer, $\mathrm{H}$ : Functional inequalities for the error function. Aequ. Math. 66(1-2), 119-127 (2003)

16. Alzer, H: Functional inequalities for the error function II. Aequ. Math. 78(1-2), 113-121 (2009)

17. Alzer, H: Error function inequalities. Adv. Comput. Math. 33(3), 349-379 (2010)

10.1186/1029-242X-2014-525

Cite this article as: Chu et al.: Best possible inequalities for the harmonic mean of error function. Journal of Inequalities and Applications 2014, 2014:525 